\title{
Bioanalysis
}

\section{The use of stable isotope-labeled drug as microtracers with conventional LC-MS/MS to support human absolute bioavailability studies: are we there yet?}

\author{
"Bioavailability assessment is an important component of new drug \\ development."
}

First draft submitted: 3 February 2016; Accepted for publication: 19 February 2016; Published online: 23 March 2016

Keywords: absolute bioavailability • AMS $\bullet$ LC-MS/MS • microtracer • stable isotope label

Bioavailability (BA) is a measurement of the rate and extent to which the active ingredient or active moiety of a drug is absorbed, reaches the systemic circulation and becomes available at the site of action. BA assessment is an important component of new drug development. Absolute bioavailability (ABA) in humans is increasingly requested by the EMEA, the US FDA and Japanese regulatory agencies for new chemical entities and is required for new drug application submission to Australian Therapeutic Goods Administration since 2006.

Traditionally, the ABA (\%F) for drugs intended for extravascular administration is obtained using a two-period crossover study design, in which an extravascular dose and an intravascular (IV) dose are administrated separately to each subject with a washout period in between. The drug concentration in circulation is then measured by traditional LC-MS/MS methods. This type of study design is often expensive and time consuming because it requires additional safety toxicology data in rodent and nonrodent for the IV route and a suitable formulation for IV dosing. Development of formulation for IV dosing at an equivalent exposure dose could be very challenging especially for poorly soluble drugs. Some estimates have put the cost and time for regulatory approval of IV administration at approximately $\$ 1.5-2$ million and taking over a year [1]. Conducting toxicology studies for IV dosing for ABA assessment has the potential for undesired safety findings that may not be relevant to the intended route of administration. In addition, the possibility of introducing variables between two dosing periods that might affect the PK data exists. ABA data from a two-way crossover study are calculated based on the assumption that drug's clearance (CL) is the same for both extravenous and intravenous administrations. However, in real clinical settings, it is very difficult to design a study that will achieve equivalent area under the curves across the two dose routes without prior knowledge of BA and volume of distribution which can only be obtained via IV dosing. As a result, apparent ABA in excess of $100 \%$ and errors in the determination of $A B A$ due to nonequivalent $\mathrm{CL}$ were reported $[1,2]$.

To overcome these drawbacks, the IV microtracer approach, first introduced in 1975 as an efficient alternative to the crossover design [3], has emerged and been widely accepted by the pharmaceutical industry as a cost-effective way to obtain ABA data in clinical studies. With this approach, subjects are administered a therapeutic dose of drug via the non-IV route, then a microtracer (either radiolabeled drug or stable isotope labeled [SIL] drug) is given via the IV route at $1 / 100$ th of therapeutic dose or less than $100 \mu \mathrm{g}$ at the expected $\mathrm{T}_{\max }$. Since the dose of the microtracer is very low, support-

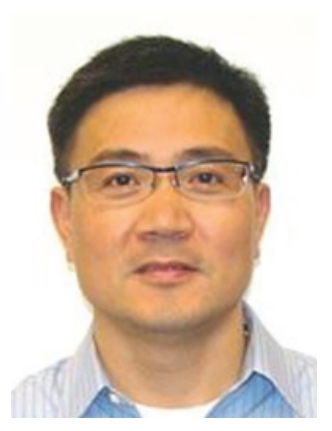

Shuguang Ma

Department of Drug Metabolism \& Pharmacokinetics, Genentech, Inc., 1 DNA Way, South San Francisco, CA 94080, USA

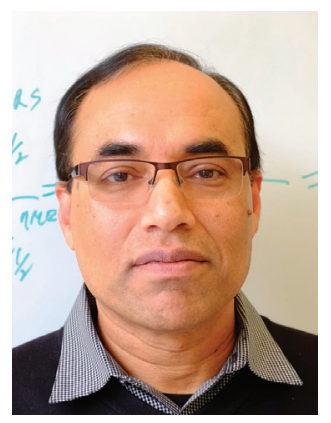

Swapan K Chowdhury Author for correspondence: Department of Drug Metabolism \& Pharmacokinetics, Millennium Pharmaceuticals, Inc., A Wholly Owned Subsidiary of Takeda Pharmaceutical Company Limited, 40 Landsdowne Street, Cambridge, MA 02139, USA swapan.chowdhury@takeda.com 
ing IV toxicology studies are not needed. Formulation development work is also limited. Even very insoluble compounds can be easily formulated with physiological concentration of saline or glucose at such a low dose. The IV microtracer is administered at the peak concentration of the non-IV route at which time the body is already behaving in the therapeutic dose range, therefore, the possibility of nonequivalent kinetics that might otherwise occur between separate dosing occasions is virtually eliminated. In addition, microtracer study with a single dosing period shortens the study duration and eliminates interoccasion variability present in crossover study design.

\section{"The technology is now ripe to face the challenges of the increased regulatory requirements to provide absolute bioavailability data for registration of pharmaceuticals..."}

When a radiolabeled drug (often ${ }^{14} \mathrm{C}$-) is used as the microtracer, accelerator MS (AMS) is required for quantifying the drug concentration. AMS is an isotope counting technique that measures the ratio of carbon isotopes $\left(\left[{ }^{14} \mathrm{C}:{ }^{12} \mathrm{C}\right]\right)$ in biological samples and offers superior sensitivity (low $\mathrm{pg} / \mathrm{ml}$ to $100 \mathrm{fg} / \mathrm{ml}$ ) over LCMS/MS [4]. The major disadvantage of AMS technology is its labor-intensive sample processing procedures. The analyte of interest must be separated and isolated from all other drug-related material by HPLC fractionation prior to AMS analysis. The sample preparation process is not amenable to automation and, thus, suffers from longer turnaround compared with the conventional LC-MS/MS analysis. The cost of AMS analysis is typically high.

SIL microtracer with LC-MS/MS analysis is an alternative, cost- and time-effective approach for supporting ABA studies if sufficiently LLOQ can be achieved to determine the drug concentrations in plasma via IV dosing. Besides LLOQ requirements, feasibility and cost of the chemical synthesis of drug labeled with multiple stable isotopes need to be considered and potentials for isotopic interferences with the LC-MS/MS detection and kinetic isotope effect on drug disposition need to be assessed before choosing stable isotope label microtracer approach $[5,6]$.

With the recent advancement of MS technologies with superior robustness, improved scan speed and ionization efficiency and enhanced ion transmission and detection, the LLOQ of SRM has been improved to low $\mathrm{pg} / \mathrm{ml}$ level with optimization of sample clean up, enrichment procedures and MS conditions [7-9]. This sensitivity makes it possible to accurately mea- sure the drug concentration in plasma following an IV administration of an SIL microtracer. The assay's sensitivity requirement is mainly determined by the dose, volume of distribution and CL of the drug. Typically, it is necessary to quantitate plasma drug concentrations for four to five half-lives to robustly characterize PK profile of a drug. For example, following a $100 \mu \mathrm{g} I V$ administration of a drug with an estimated volume of distribution of 1001 in humans, the drug concentration is calculated to be $31.3 \mathrm{pg} / \mathrm{ml}$ at $5 \times \mathrm{t}_{1 / 2}$. So, the assay with an LLOQ at $10-20 \mathrm{pg} / \mathrm{ml}$ would be necessary. Allometry (animal-to-human scaling methods), semiphysiological methods and full physiologically based PK models have also been developed for predicting IV PK parameters and concentration-time profile shape [10], which can provide $\mathrm{C}_{\min }$ requirement of the LC-MS/MS assay development.

Due to improvement in LC-MS technologies it is becoming clear that as an alternative to AMS, conventional LC-MS/MS is going to play an important role in microtracer studies. Most recently, four (daclatasvir [6], tofogliflozin [11], evacetrapib [12] and ibrutinib [13]) ABA studies published in the literature have used SIL drug as microtracer. The ABA of daclatasvir was determined in healthy subjects who received a $60 \mathrm{mg}$ tablet orally followed by a $100 \mu \mathrm{g}\left[{ }^{13} \mathrm{C}_{2},{ }^{15} \mathrm{~N}_{4}\right]-$ daclatasvir intravenous dose. The LC-MS/MS SRM method using ${ }^{13} \mathrm{C}_{10}$-daclatasvir as internal standard (IS) had an LLOQ of $20 \mathrm{pg} / \mathrm{ml}$. The accuracy and precision was sufficient to cover analysis up to $72 \mathrm{~h}(>5$ half-lives). In this comprehensive study, the authors initially evaluated isotope contribution to SIL and the IS using theoretical isotope distribution pattern analysis followed by an in vivo study in the monkeys to assure complete lack of isotopic interference. Using a novel duo-tracer approach, Schwab et al. [11] combined a traditional absorption, distribution, metabolism and excretion study with ABA determination. In this study six healthy subjects received $20 \mathrm{mg}\left[{ }^{12} \mathrm{C} /{ }^{14} \mathrm{C}\right]$-tofogliflozin orally and a concomitant $100 \mu \mathrm{g}\left[{ }^{13} \mathrm{C}_{6}\right]$-tofogliflozin intravenously. The ${ }^{13} \mathrm{C}$-drug was quantified by LC-MS/MS in the concentration range of $10 \mathrm{pg} / \mathrm{ml}$ to $100 \mathrm{ng} / \mathrm{ml}$ using a $\mathrm{D}_{4}$-hydroxyl-metabolite as internal standard. This LC-MS/MS method was able to quantify plasma drug concentration up to $24 \mathrm{~h}$ postdose ( $\sim 5$ half-lives). This study represents a novel concept in getting total absorption, distribution, metabolism and excretion data in a single, one-period study. More recently, the ABA of evacetrapib [12] and ibrutinib [13] was determined in healthy subjects by administration of $130 \mathrm{mg}$ evacetrapib orally and $175 \mu \mathrm{g}\left[{ }^{13} \mathrm{C}_{8}\right]$ evacetrapib intravenously and $560 \mathrm{mg}$ ibrutinib orally and $100 \mu \mathrm{g}\left[{ }^{13} \mathrm{C}_{6}\right]$-ibrutinib intravenously, respectively. In both cases, LC-MS/MS methods were developed 
and fully validated to quantify $\left[{ }^{13} \mathrm{C}_{8}\right]$-evacetrapib and $\left[{ }^{13} \mathrm{C}_{6}\right]$-ibrutinib with sufficiently LLOQ to cover up to at least $4 \times$ of $t_{1 / 2}$. These examples that required the LLOQ in the range of $2-10 \mathrm{pg} / \mathrm{ml}$ demonstrated that LC-MS/MS can be a practical approach to support ABA studies with SIL microtracers.

With the continuous improvement in the sensitivity of the MS instrumentation, we believe more and more ABA studies will be conducted using SIL microtracer with LC-MS/MS analysis. This strategy significantly reduces time, costs and resources, compared with twostage traditional ABA studies or the radiomicrotracer with accelerator MS. Using the SIL approach, drug concentration from both the extravascular route of administration at therapeutic dose and the IV at microdose can be determined from the analysis of a single set of plasma samples with the same LC-MS/MS run.

In conclusion, the microtracer approach has opened up and simplified the clinical study design to obtain

\section{References}

1 Lappin G. Approaches to intravenous clinical pharmacokinetics: recent developments with isotopic microtracers. J. Clin. Pharmacol. 56(1), 11-23 (2016).

2 Ward KW, Hardy LB, Kehler JR, Azzarano LM, Smith BR. Apparent absolute oral bioavailability in excess of $100 \%$ for a vitronectin receptor antagonist (SB-265123) in rat. II. Studies implicating transporter-mediated intestinal secretion. Xenobiotica 34(4), 367-377 (2004).

3 Strong JM, Dutcher JS, Lee WK, Atkinson AJ Jr. Absolute bioavailability in man of $\mathrm{N}$-acetylprocainamide determined by a novel stable isotope method. Clin. Pharmacol. Ther. 18(5 Pt 1), 613-622 (1975).

4 Seymour MA. Accelerator MS: its role as a frontline bioanalytical technique. Bioanalysis 3(24), 2817-2823 (2011).

5 Xu XS, Jiang H, Christopher LJ, Shen JX, Zeng J, Arnold ME. Sensitivity-based analytical approaches to support human absolute bioavailability studies. Bioanalysis 6(4), 497-504 (2014).

6 Jiang H, Zeng J, Li W et al. Practical and efficient strategy for evaluating oral absolute bioavailability with an intravenous microdose of a stable isotopically-labeled drug using a selected reaction monitoring mass spectrometry assay. Anal. Chem. 84(22), 10031-10037 (2012).

7 Keski-Rahkonen P, Desai R, Jimenez M, Harwood DT, Handelsman DJ. Measurement of estradiol in human serum
ABA data. Nowadays, LC-MS/MS assays are commonly approaching to the detection limit of low $\mathrm{pg} /$ $\mathrm{ml}$, which makes it the preferred bioanalytical tool of choice for supporting SIL microtracer absolute BA studies due to its simplicity, robustness, high-throughput analysis, time and cost-effectiveness and superior selectivity. The technology is now ripe to face the challenges of the increased regulatory requirements to provide ABA data for registration of pharmaceuticals.

\section{Financial \& competing interests disclosure}

The authors have no relevant affiliations or financial involvement with any organization or entity with a financial interest in or financial conflict with the subject matter or materials discussed in the manuscript. This includes employment, consultancies, honoraria, stock ownership or options, expert testimony, grants or patents received or pending, or royalties.

No writing assistance was utilized in the production of this manuscript.

by LC-MS/MS using a novel estrogen-specific derivatization reagent. Anal. Chem. 87(14), 7180-7186 (2015).

8 Wieder ME, Paine SW, Hincks PR, Pearce CM, Scarth J, Hillyer L. Detection and pharmacokinetics of salbutamol in thoroughbred racehorses following inhaled administration. J. Vet. Pharmacol. Ther. 38(1), 41-47 (2015).

9 Aubry AF. LC-MS/MS bioanalytical challenge: ultra-high sensitivity assays. Bioanalysis 3(16), 1819-1825 (2011).

10 Jones HM, Dickins M, Youdim K et al. Application of PBPK modelling in drug discovery and development at Pfizer. Xenobiotica 42(1), 94-106 (2012).

11 Schwab D, Portron A, Backholer Z, Lausecker B, Kawashima K. A novel double-tracer technique to characterize absorption, distribution, metabolism and excretion (ADME) of $\left[{ }^{14} \mathrm{C}\right]$ tofogliflozin after oral administration and concomitant intravenous microdose administration of $\left[{ }^{13} \mathrm{C}\right]$ tofogliflozin in humans. Clin. Pharmacokinet. 52(6), 463-473 (2013).

12 Cannady EA, Aburub A, Ward C et al. Absolute bioavailability of evacetrapib in healthy subjects determined by simultaneous administration of oral evacetrapib and intravenous $\left[{ }^{13} \mathrm{C}_{8}\right]$-evacetrapib as a tracer. J. Labelled Comp. Radiopharm. doi:10.1002/jlcr.3358 (2015) (Epub ahead of print).

13 De Vries R, Smit JW, Hellemans P et al. Stable isotopeintravenous microdose for absolute bioavailability and effect of grapefruit juice on ibrutinib in healthy adults. Br. J. Clin. Pharmacol. 81(2), 235-245 (2015). 